\title{
Research on Anti-Tax Evasion System based on Union-Bank Online Payment mode
}

\author{
Yang Qifeng ${ }^{1}$, Feng Bin ${ }^{2}$, and Song Ping ${ }^{3}$ \\ 1-3 Economics College of Wuhan University of Technology, \\ Wuhan 430070, Hubei.P.R, China \\ Email:1 yangqifengwhut@163.com, 213027154642@vip.163.com, \\ songpingwhut@163.com
}

\begin{abstract}
The popularity of the e-commerce facilitates the tax evasion a lot, and the anti-tax evasion work in the e-commerce area becomes urgent. We introduced a union-bank online payment mode, which can solve many problems of existing mode such as main body status, standardization and inter-bank payment. Besides, it provides a platform for some value added services such as anti-tax evasion, anti-money laundering, credit evaluation, etc. In this mode, the transaction data is centralized so that we can carry on the investigation during the online payment process. Based on this, we designed an anti-tax evasion system utilizing the data mining, OLAP and data warehouse technologies. This system can monitor the transaction dynamically and judge the tax evasion behavior by analyzing abundant transaction data according to relevant tax law, and the knowledge in the knowledge base and expert base. Beside, it collects the tax evasion rule as knowledge in order to update itself automatically.
\end{abstract}

\section{Introduction}

The rapid development of e-commerce improves the flourish of the world economy and rational allocation of the worldwide resource. Meanwhile, it changes the traditional transaction mode and brings big challenge to the tax system. In the virtual market of the e-commerce, the customers can be anonymous and the sellers can conceal their address. Even the transaction can be finished without any record, and this led to many tax evasion phenomena by ecommerce.

The reason why e-commerce facilitates the tax evasion is various. Firstly, the characteristic of e-commerce makes the transaction itself difficult to be monitored. The e-commerce activities need no fixed shop, paper contract that is necessary in traditional transaction, and the goods can be immaterial such as digital product. All these characteristic will place a premium on tax evasion [1]. Secondly, the tax law of e-commerce is distempered. The problem that should all 
the e-commerce activities pay the tax puzzled many countries for a long time. Some countries suggested part tax of the transaction by e-commerce should be avoided while other countries didn't think so. There is no agreement on how to impose the tax of the transaction by e-commerce. Thirdly, the revenue management of the e-commerce is disordered [2]. At present, many sellers on the net haven't registered in the administration for industry and commerce, and have no business license. This adds the difficulty of the taxation. Especially, the online payment is preferred to by the tax dodger because it reduces the cost and risk.

To solve the problem of tax evasion, we need to build an effective AntiTax Evasion (ATE) system with effective management mechanism. The unionbank online payment mode can provide such platform for anti-tax evasion work. On such platform, the transaction data of the whole country through online payment can be centralized, on which we can carry on the data mining so as to find out the tax evasion behavior. Based on this mode, we designed an anti-tax evasion system using data mining technology, which can improve the ability to find out tax evasion behavior a lot during the online payment process.

\section{Union-bank online payment mode}

\subsection{Advantage of the union-bank online payment mode}

Nowadays, the existing online payment mode in our country can't meet the need of favorable development of e-commerce, it's impossible to become the leading online payment mode: 1) the third party online payment platform is questioned in its main body status and business scope; the prestige of itself is not enough; and the problem of fund in float may cause system risk. So it's impossible to become the leading mode.[3,4] 2) The commercial banks can provide online payment by online bank gateway, but they all do things in their own way in the transaction procedure, interface standard and authentication mechanism. This increases the difficulty of each side and led to waste resource because of the development of multi-payment gateway and interface software. The data of information flow, capital and logistics flow referred during the e-commerce process is not normative and unified. The most pivotal issue is the distribution of benefit; it's impossible to build up the leading online payment mode based on certain commercial bank. 3) The China UnionPay is a third party online Payment Company in essence, however it succeeded in credit card electronic payment area, also faced the same problem as the third party online payment company. In this situation, it's impossible to provide the platform which can centralize the transaction data for many value added service including ATE, Anti-Money Laundering (AML), credit evaluation, etc.

The e-commerce online payment in our country faced the challenge of main body status, standardization, innovative service and inter-bank payment, so it's necessary to build up the leading online payment mode with Chinese characteristics. The payment flow chart of Union-Bank online payment mode introduced in this paper is shown in fig 1. This mode can overcome the deficiency of the existing mode, and promote the well-liking development of ecommerce by continuous innovation. 


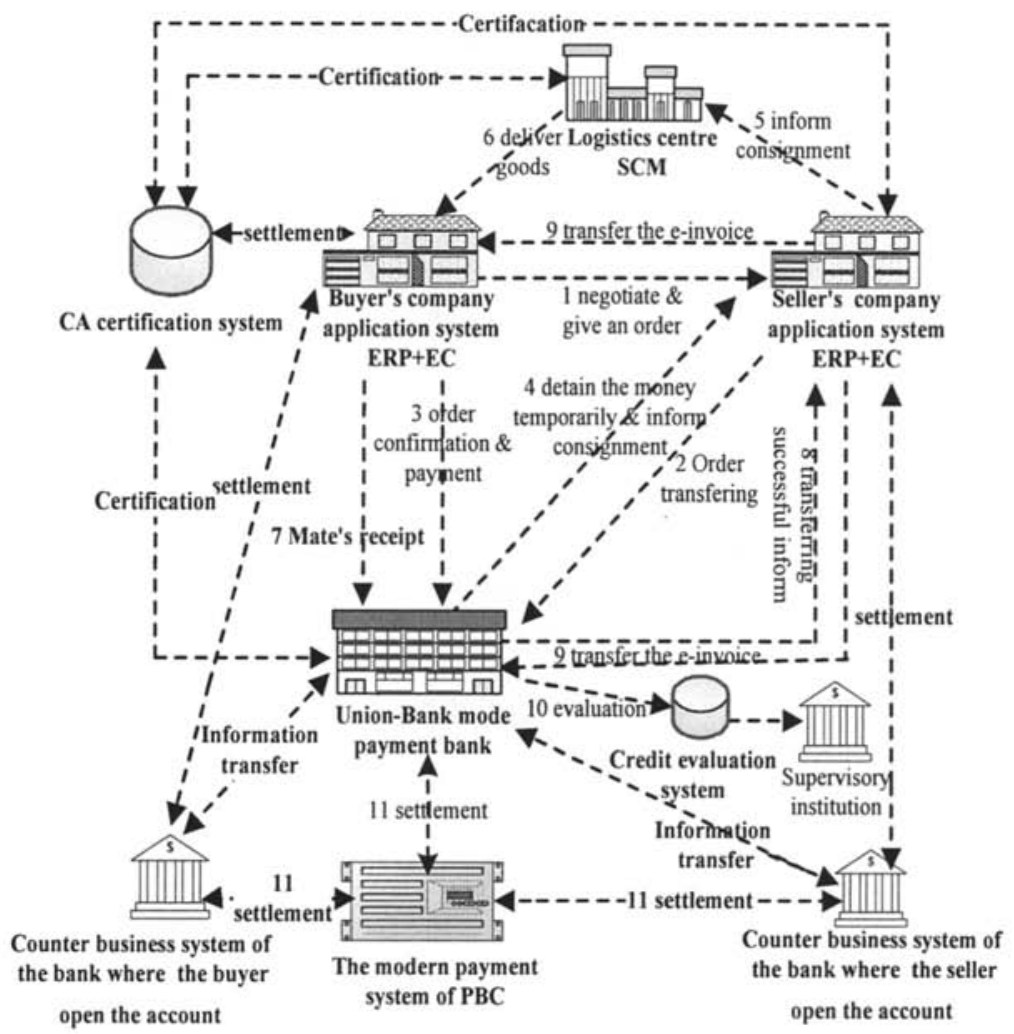

Fig. 7. Payment flow chart of the Union-Bank mode

\subsection{Process of the union-bank online payment mode}

The union-bank online payment mode we discussed is able to become leading mode that is just needed in our country. By the integrative connection of China modern payment system of PBC to each member commercial bank, the application system, payment gateway, network point resource and client resource can be shared, and the inter-bank payment service can be realized favorably by the extension of the payment supply chain. In this mode, the system of relevant institutions, such as buyer, seller, certification centre, logistics centre, modern payment system of $\mathrm{PBC}$, mobile communication system, 315 customer association, quality surveillance office, industry and commerce administrative bureau, police bureau, can be connected and work collaboratively with unified online payment gateway, information interface standard and normal processing procedure. This will solve the chock point which blocks the development of the e-commerce effectively. By constituting national concentrating e-commerce basic data resource base building upon union-bank mode, this study takes the achievement in relevant area of domestic and international researches for reference, colligates the fruit of computer science, operations research, industry project, commercial strategy, management science, law science and so on, studies the intelligent recommend technology base on the users experience, case consequence, knowledge noumenon, prediction and early warning method, CRM, 
SCM and credit evaluation method, and the innovative service regarding the online payment as hinge, in order to provide support of theory and method for the development of e-commerce economy, and create value for the online payment supplier and client.

The UBOP bank uses the "head office-branch" structure, and the UBOP platform is the national data concentration system. Participates in B2B of UBOP include: 1) Buyer's company (ERP+EC). Its main factors include goods purchase, payment, and e-check; 2) Seller's company (ERP+EC). Its main factors include goods delivery, collection, and transfer of the e-invoice; 3) Certificate Authority. It takes the charge of inspecting the legitimacy of the public key as a believed third party during the e-commerce; 4) Logistics centre. It carries on the goods delivery according to seller's order, and participate in the supply chain management; 5) UBOP centre (bank). It's the core of the online payment, and it's a special bank engaging online payment service that can take part in the settlement of PBC. Its main function is payment: both sides of the transaction should open an account which can be connected to their account in commercial bank before the online business, and then use it pay or collect money. Their extensive services include: supply chain return, quality testing complains, credit mechanism construction, etc. 6) bank where the buyer/seller open the account. The account in the commercial bank where the buyer/seller opens the account associates with the account in UBOP, and the money can be transferred between them; 7) prestige evaluation institution. It evaluates the prestige of the both parties of the transaction, Logistics Company and so on which participate in the e-commerce. This institution associates with the national quality testing department, consumers' association, and arbitration department.

\section{Logical framework of ATE service system}

Based on the online payment mode, the logical framework of the ATE service system can be divided into five layers: database layer, basic data resource base layer, data analysis layer, application service layer and interface layer as shown in the figure 3.1 .

(1) Database layer: It is composed of abundant basic business data including user data, transaction data, tax imposition data and a history database. These provide the data support for the basic data resource layer and real-time ATE intelligent analysis and they are updated by the data import from the unionbank centre.

User data refers to relevant data of the company or customer, including the register information (register identity, register time, company or customer detail introduction, encrypted account information), use information (use time, use IP address and use aim) and credit information (times of successful transaction, times being complaint about and the credit evaluation of both sides of the transaction and union-bank). 


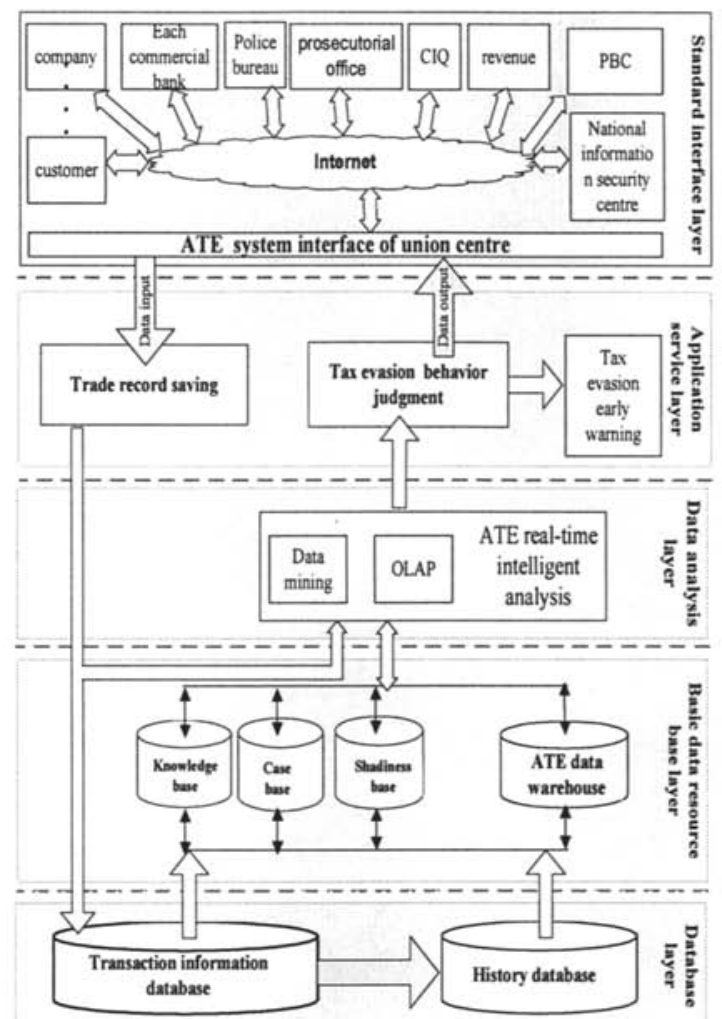

Fig.2. Logical framework of the ATE system

Transaction data includes the data relating to the transaction between the users which compounds of the transaction content (bargainers of both sides, goods information, price, paying time and paying amount), transaction process (bargain record, order of the payment and goods delivering, banks of opening account of both sides, order and invoice) and transaction result (successful or failing, failure reason, loss by the failure, complaint and the process result).

Tax imposition data refers to the relevant tax information about the transaction. It includes the transaction type, goods type, tax type, tax amount that should impose on and relevant laws.

History database is used for storing the historical data of the user information database, trade information database and capital flow database. Because these three databases will be cleaned up maybe every year, the data will be stored in history database. Certainly the history database will be clean up too, but, every 3 or 5 years. The history data finally will be sent to ATE data warehouse. Given the large amount of the information referred in ATE area, we construct the history database as a temporary buffer memory between database layer and ATE data warehouse.

(2) Basic data resource base layer: This layer stores some knowledge for data mining and suspicious transaction. By data mining, it analyzes the relation among the data from database layer so as to find some laws of these data and 
provide support for the data analysis layer. It includes knowledge base, case base and shadiness base.

Knowledge Base: $\mathrm{KB}$ is based on the expert experience and booklore, and refreshed dynamically by the connection to the database. The basic structure of $\mathrm{KB}$ is hiberarchy. Its lowest layer is "fact knowledge" such as theoretical knowledge and factual data of the tax evasion, the middle layer is knowledge which controls the "fact knowledge" such as some rules and processes of the tax evasion, and the highest layer is "strategy" which controls the middle layer which we call "ATE strategies". The ATE knowledge base aims at the tax evasion area and collects the solution so as to realize the intelligence of the system.

Case Base: It collects the case in the tax evasion and ATE area, analyses the relation between them in order to extract the tax evasion methods and their representations. This will be helpful for ATE real-time intelligent analysis to judge if the current case matches the characteristic from the case base.

Shadiness Base: This stores the knowledge of the suspicious trade which can learn, adapt and update itself. The real-time intelligent analysis will obtain relevant cue from the shadiness base, and if it finds some suspicious trade, it will send a feedback report to the shadiness base.

ATE Data warehouse: The data in the database layer is independent and is classified into certain kind of information. However, the relation of them can't be reflected. Although the history database stores all the information of the three databases, it just keeps the independent structural data and won't analyze its rule. The ATE data warehouse is data integration factory oriented ATE area for decision supporting of ATE real-time intelligent analysis. All the information from database layer will flow into this warehouse and is processed here.

(3) Data analysis layer: The main function of this layer is to analyze the data form database and basic data resource base layer utilizing data mining technology. The ATE real-time analysis cleans up the data from database and basic data resource base layer, format and filtrate it through data mining, and then analyzes the feedback information and output the result to the union-bank centre. This layer clean up and convert the allopatric distributed data resource (include the database of each platform, text file, HTML file, knowledge base and so on) according to the subject heading list definition, data resource definition and data extract rule definition. Then reorganize and process the data and load them to the aim base in the data warehouse. The real-time intelligent analysis includes two modules which are OLAP (Online Analytical Processing) and Data mining. Utilizing these two powerful tools, the analysis can extract the useful information from the data for decision supporting.

Because it's necessary to carry on the operation such as Slice, Dice, Roll-up, Drill-down during the application of OLAP, we choose the cube data mode to describe the structure of multidimensional data. For instance, we can construct the cube data mode as figure 3 shows to describe the tax information. This mode is composed of one fact table and four dimensional tables. The fact table shows the tax paying situation of certain people in certain transaction and certain time. The taxpayer dimensional table lists the basic information of the taxpayer; the tax dimensional table shows the relevant tax information; data dimensional table refers to the time of the data using the combination of year, month and day; the transaction dimensional table lists the relevant transaction information corresponding to the tax. Thus these four dimensional table make up of the 
mode

Hypercube data mode, and we can carry on data analysis deeply from multi angle by this mode.

Data mining is another useful tool which is complementary with the OLAP. It carries on the knowledge discovery on the data in the data warehouse by AI and statistics analysis technology, and provides the knowledge for the user in the form of model as the foundation of tax evasion behavior judgment. Before the application of the models, we can validate them by OLAP. For example, we can construct the credit evaluation model of the taxpayer to strength the tax imposition, and construct the tax imposition elastic analysis and income prediction of the taxpayer model to make out the tax imposition task. And then validate the fitting degree of the models by OLAP.

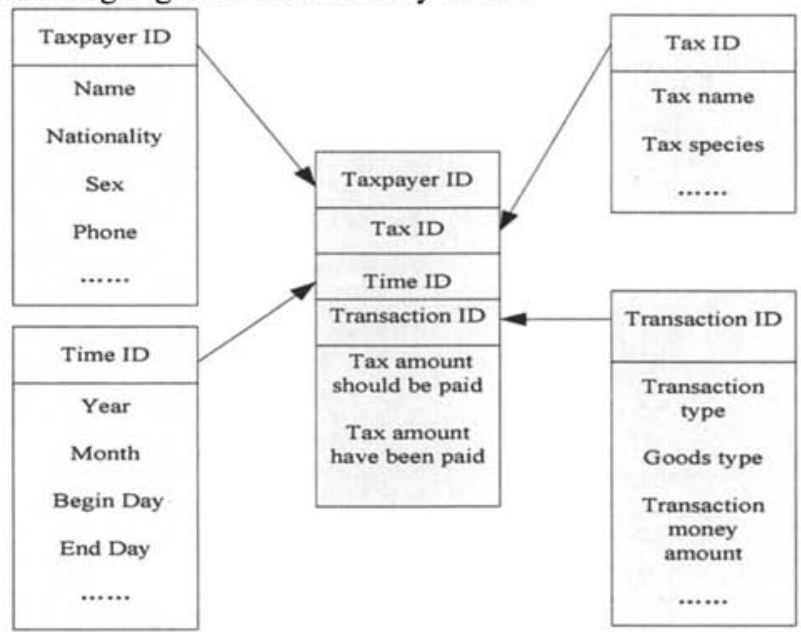

Fig.3. Tax paying information hypercube mode

(4) Application service layer: This layer mainly includes two function services: trade record saving and legality judgment of the trade behavior. When receiving the data from union-bank centre, application service layer will extract relevant information from the data and format it according to the standard format of the database, and then output it to each database. When receiving the result from data analysis layer, application service layer will display the information to the user terminal and request a decision if the result is ambiguous, and send an alarm to early warning centre if the tax evasion is ascertained. The final result is output to the union-bank centre.

(5) Standard Interface layer: This layer is the external interface integration. The trade information from the company or customer transmits to the union-bank center through Internet, and then to the ATE service system. Likewise, the ATE service system uses the interface of union-bank centre to transmit the result to $\mathrm{PBC}$ and National information security centre. When there is a tax evasion behavior, the ATE service system will cooperate with the other institutions and transmit information to there using this interface. 


\section{Key technologies}

Data warehouse is the basis of the data mining and is used for supporting the decision management It is a data aggregation which is subject oriented, integrative, steady and changing continuously as the time. Compare with the traditional database that only include current fixed data mode, the data warehouse is an integrative method of distributed heterogeneous data system. It carries on the summarization and clustering of the data which is structured, halfstructured, non-structured and with different source, extracts the decision supporting oriented part and loads it to the data warehouse. Thus, we can realize the uniform management of the data, and access data warehouse directly when inquiring without the need of accessing other information resource. By this, what the data mining faced is the uniform data arranged, and the processing becomes easier.

OLAP combines the concept of multidimensional database and multidimensional analysis, enables the analyzer, administer and the executer to access the information fast, conformably and interactively. The core of the OLAP is "multidimensional". For example, generally the revenue administration will consider the tax income from many aspects such as time, district and tax species which just are the "dimension". Each combination of these dimension and the tax income compose of the multidimensional array that is the basis of the OLAP analysis, and can be denoted as time, district, tax species and tax income. By all kinds of analytic action to the multidimensional array such as Slice, Dice, Pivot, Drill- down and Roll-up, the OLAP enable the decision maker to observe the data in the database from multi-angle so as to deeply understand the information hidden in the data.

Data mining is a method of extracting hidden, useful information from abundant of data utilizing artificial intelligence, advanced statistics methods, and technologies such as cluster analysis, neural net, data visualization and decision tree. [5] This information can open out the unapparent mode, trend and rule of data. The ATE system can analyze the existing data fully by data mining in order to provide evaluation and prediction. Besides, it can offer the leader layer fully decision foundation by understanding the basic information and the change of each tax resource, the influence of the main economic index to the tax resource in each district, transaction type, goods type and the tax amount that should be paid and so on.

\section{Conclusion}

In this paper, we introduced the union-bank online payment mode in which the transaction data is centralized at each level. Based on this platform, we designed an anti-tax evasion system as a value added service of the union-bank center. This system is able to find out the abnormal situation of the tax paying using the data mining, OLAP and data warehouse technologies during the online payment process. These are meaningful to the tax paying in the e-commerce area and strength the management of online transaction. We hope relevant criterion and law will come on fast so that the tax management of the e-commerce can have a foundation and direct to rely on. 
mode

\section{Acknowledgements}

This Research was supported by the Doctor Fund of Wuhan University of Technology under Grant 471-38650316 and the graduate student teaching reform Fund of Wuhan University of Technology under Grant 200404.

\section{Reference:}

1. Song Xiaozhong, Sun Jisheng, Suggestion to the ATE of E-Commerce in China, Tax imposition, 2006, No.4, pp: 9-15

2. Luo Qianzhang, Analysis of Borderline of Tax Evasion and Tax Avoidance in E-Commerce, Economic \& Trade Update, Vol.4, No.49, Nov.2006

3. Zhou Xiaofan, Wu Wei, The Third Party Online Payment Platform is difficult to become popular, Economic forum, Dec.2006

4. Xin Yunyong, Hu Xiaohua, Online payment duplicates China Uionpay. Internet Weekly, Mar. 2005

5. Wang Tao, Xu Ping, Design of Tax Decision Supporting System based on Data Mining, Management Sciences in China, Vol.16, No.1, Feb, 2003

6. Qiu Ling, Discussion of ATE management in e-commerce, Journal of Guangdong Vocational College of Finance and Economics, No.3, 2002

7. Ran Chunyu, Gu Chuan, Chen Min, Huang Shaoluan, Design of data warehouse of tax DSS system, Computer Applications, No.S1, 2005

8. Fang Baorong, Wang Lei, Discussion of computer tax monitoring and control system design, Taxation research journal, No.06, 2000

9. Chen Dan, Mode design of tax imposition system, Shang Hai accountant, No.6, 2003 